\title{
Note: Correction to the 1997 Tutorial on Reed-Solomon Coding
}

\author{
James S. Plank $\quad$ Ying Ding \\ University of Tennessee \\ Knoxville, TN 37996 \\ [plank, ying] @es.utk.edu \\ Technical Report UT-CS-03-504 \\ Department of Computer Science \\ University of Tennessee \\ April 24, 2003
}

\section{Introduction}

In 1997, SPE published a tutorial by Plank [19] on implementing Reed-Solomon codes for erasure correction in redundant data storage systems. The motivation of this tutorial was to present these codes, which are typically described mathematically by coding theorists, in a way accessible to the programmers who need to implement them. The tutorial as published presented an information dispersal matrix $A$, which does not have the properties claimed - that the deletion of any $m$ rows results in an invertable $n \times n$ matrix. The purpose of this note is to present a correct information dispersal matrix that has the desired properties, and to put the work in current context.

\section{The Continued Need For Erasure Correcting Codes}

As disk array technology continued to blossom in the 1990's [4, 5], a need arose to tolerate a disk's failure without waiting for the disk to be repaired. Straight replication performs this fault-tolerance, but at a high storage overhead. RAID Level 5 encoding, termed "N+1 Parity" [5], reduces the storage overhead for fault-tolerance, and allows a parity disk to store redundancy for $n$ data disks in such a way that the failure of any single disk may be tolerated. However, as the number of disks in a disk array grows, so does the the need to tolerate multiple simultaneous failures. ReedSolomon coding has the properties necessary to add arbitrary levels of fault-tolerance to disk array systems. One may add $m$ coding disks to $n$ data disks so that the failure of any $m$ disks may be tolerated, and although none of the levels of RAID employs Reed-Solomon codes, the original work on disk arrays make note of the codes' desirable properties [5]. 
As wide-area network computing has become more popular, the uses of erasure-correcting codes have broadened. Rizzo employs them to avoid retransmission in point-to-point [20] and multicast [21] communication protocols. This work has resulted in standardization efforts for such codes in multicast scenarios from the IETF [15, 16]. Additional uses of Reed-Solomon codes have been in cryptography [8], distributed data structures [10], energy-efficient wireless communication [7] and distributed checkpointing [18].

The advent of wide-area and peer-to-peer storage systems has further motivated the need for erasure-correcting codes. For example, OceanStore employs Reed-Solomon coding for RAID-like fault-tolerance in a wide-area file system [9]. More interestingly, several content dispersal systems have noted that erasure coding can be used for caching rather than for fault-tolerance [2, 3, 22]. Specifically, suppose that $n$ blocks of a file need to be stored in a wide-area storage substrate, so that clients in all parts of the network may access it. With replication, clients must find the closest copies of each of the $n$ blocks in order to retrieve the file. However, with erasure-correcting codes, $m$ extra coding blocks may be distributed with the $n$ blocks of the file so that each client need only retrieve the $n$ closest blocks in order to reconstruct the file. As files grow in size, the power of this application will be immense; hence the need to correct the error of the 1997 Reed-Solomon coding tutorial.

There are other erasure coding techniques in addition to the one which this tutorial addresses. Examples are Tornado codes [13, 14], Cauchy Reed-Solomon codes [1] and other parity-based schemes [6]. Of these, Tornado codes are worth special mention, as they form the backbone of the Digital Fountain content dispersal system [2]. Tornado codes have a randomized structure so that with the addition of $m$ extra parity blocks, a file may be reconstructed from any $n+\epsilon$ blocks. The randomized structure ensures that $\epsilon$ should be small. In a performance evaluation conducted by Luby [12], Tornado codes display significantly better encoding and decoding performance than the codes in this paper (termed "Vandermonde-based Reed-Solomon codes") for large data sizes and large values of $m$. For small values of $m$, a true comparison has yet to be performed. Currently, there is no implementation guide for Tornado codes akin to [19]. As the knowledge of their performance advantages become more widespread, perhaps this will change.

\section{A Correct Information Dispersal Matrix $B$}

The desired properties for the information dispersal matrix for Reed-Solomon coding is that:

- It is an $(n+m) \times n$ matrix.

- The $n \times n$ matrix in the first $n$ rows are the identity matrix.

- Any submatrix formed by deleting $m$ rows of the matrix is invertible.

We denote the correct information matrix $B . B$ is derived from an $(n+m) \times n$ Vandermonde matrix using a sequence of elementary matrix transformations:

1. Any column $C_{i}$ may be swapped with column $C_{j}$. 
2. Any column $C_{i}$ may be replaced by $C_{i} * c$, where $c \neq 0$.

3. Any column $C_{i}$ may be replaced by adding a multiple of another column to it: $C_{i}=C_{i}+c * C_{j}$, where $j \neq i$ and $c \neq 0$. Since arithmetic is over a Galois field, the addition operation is bitwise exclusive-or.

The $i, j$-th element of a Vandermonde matrix is defined to be $i^{j}$ :

$$
\left[\begin{array}{ccccc}
0^{0}(=1) & 0^{1}(=0) & 0^{2}(=0) & \ldots & 0^{n-1}(=0) \\
1^{0} & 1^{1} & 1^{2} & \ldots & 1^{n-1} \\
2^{0} & 2^{1} & 2^{2} & \ldots & 2^{n-1} \\
\vdots & \vdots & \vdots & & \vdots \\
(n+m-1)^{0} & (n+m-1)^{1} & (n+m-1)^{2} & \ldots & (n+m-1)^{n-1}
\end{array}\right]
$$

By definition, this matrix has the property that any submatrix formed by deleting $m$ rows of this matrix is invertible [17]. Moreover, any matrix derived from this matrix by a sequence of elementary matrix transformations maintains this property (since elementary matrix operations do not change the rank of a matrix [11]). Therefore, constructing the matrix $B$ is a simple matter of performing elementary transformations on the Vandermonde matrix until the first $n$ rows are the identity matrix.

The algorithm for doing constructing $B$ is as follows:

- Suppose the first $i-1$ rows of the matrix are identity rows, and $i<n$. At each step, we will turn row $i$ into an identity row, without altering the other identity rows. If the $i$-th element of row $i$ is equal to zero, find a column $j$ such that $j>i$ and the $j$-th element of row $i$ is non-zero, and swap columns $i$ and $j$. Such a column is guaranteed to exist; otherwise the first $n$ rows of the matrix would not compose an invertible matrix. Moreover, since $j>i$, swapping columns $i$ and $j$ will not alter the first $i-1$ rows of the matrix.

- Let $f_{i, i}$ be the value of the $i$-th element of row $i$. Let $f_{i, i}^{-1}$ be the multiplicative inverse $f_{i, i}$. In other words, $f_{i, i} * f_{i, i}^{-1}=1$. Since $f_{i, i} \neq 0, f_{i, i}^{-1}$ is guaranteed to exist. if $f_{i, i} \neq 1$, replace column $C_{i}$ with $f^{-1} i, i * C_{i}$.

- Now $f_{i, i}=1$. For all columns $j \neq i$ and $f_{i, j} \neq 0$, replace column $C_{j}$ with $C_{j}-f_{i, j} C_{i}$, where $f_{i, j}$ is the $j$-th element in row $i$. At the end of this step, rows 0 through $i$ are identity rows, and the matrix still has the property that the deletion of any $m$ rows yields an invertible matrix.

- Repeat this process until the first $n$ rows are identity rows, and the construction of $B$ is complete.

\section{Example}

As an example, we construct $B$ for $n=3, m=3$, over $\mathrm{GF}\left(2^{4}\right)$. As detailed in [19], in $\mathrm{GF}\left(2^{4}\right)$, addition is performed by exclusive-or, and multiplication/division may be performed using logarithm tables, reproduced in Table 1 .

The $6 \times 3$ Vandermonde matrix over $G F\left(2^{4}\right)$ is: 


\begin{tabular}{|c||c|c|c|c|c|c|c|c|c|c|c|c|c|c|c|c|}
\hline$i$ & 0 & 1 & 2 & 3 & 4 & 5 & 6 & 7 & 8 & 9 & 10 & 11 & 12 & 13 & 14 & 15 \\
\hline gflog $[\mathrm{i}]$ & - & 0 & 1 & 4 & 2 & 8 & 5 & 10 & 3 & 14 & 9 & 7 & 6 & 13 & 11 & 12 \\
\hline gfilog $[\mathrm{i}]$ & 1 & 2 & 4 & 8 & 3 & 6 & 12 & 11 & 5 & 10 & 7 & 14 & 15 & 13 & 9 & - \\
\hline
\end{tabular}

Table 1: Logarithm tables for $G F\left(2^{4}\right)$

$$
\left[\begin{array}{lll}
0^{0} & 0^{1} & 0^{2} \\
1^{0} & 1^{1} & 1^{2} \\
2^{0} & 2^{1} & 2^{2} \\
3^{0} & 3^{1} & 3^{2} \\
4^{0} & 4^{1} & 4^{2} \\
5^{0} & 5^{1} & 5^{2}
\end{array}\right]=\left[\begin{array}{lll}
1 & 0 & 0 \\
1 & 1 & 1 \\
1 & 2 & 4 \\
1 & 3 & 5 \\
1 & 4 & 3 \\
1 & 5 & 2
\end{array}\right]
$$

Row 0 is already an identity row. To convert row 1 , we note that $f_{1,0}=f_{1,1}=f_{1,2}=1$, so we need to replace $C_{0}$ with $\left(C_{0}-C_{1}\right)$ and $C_{2}$ with $\left(C_{2}-C_{1}\right)$. The resulting matrix is:

$$
\left[\begin{array}{lll}
1 & 0 & 0 \\
0 & 1 & 0 \\
3 & 2 & 6 \\
2 & 3 & 6 \\
5 & 4 & 7 \\
4 & 5 & 7
\end{array}\right]
$$

All that is left is to convert row 2. First, since $f_{2,2} \neq 1$, we need to replace $C_{2}$ with $6^{-1} C_{2}=7 C_{2}$ :

$$
\left[\begin{array}{lll}
1 & 0 & 0 \\
0 & 1 & 0 \\
3 & 2 & 1 \\
2 & 3 & 1 \\
5 & 4 & 6 \\
4 & 5 & 6
\end{array}\right]
$$

Then we replace $C_{0}$ with $\left(C_{0}-3 C_{2}\right)$ and $C_{1}$ with $\left(C_{1}-2 C_{2}\right)$ to yield our desired $B$ :

$$
\left[\begin{array}{ccc}
1 & 0 & 0 \\
0 & 1 & 0 \\
0 & 0 & 1 \\
1 & 1 & 1 \\
15 & 8 & 6 \\
14 & 9 & 6
\end{array}\right]
$$




\section{References}

[1] J. Blomer, M. Kalfane, M. Karpinski, R. Karp, M. Luby, and D. Zuckerman. An XOR-based erasure-resilient coding scheme. Technical Report TR-95-048, International Computer Science Institute, August 1995.

[2] J. Byers, M. Luby, M. Mitzenmacher, and A. Rege. A digital fountain approach to reliable distribution of bulk data. In ACM SIGCOMM '98, pages 56-67, Vancouver, August 1998.

[3] J. W. Byers, M. Luby, and M. Mitzenmacher. Accessing multiple mirror sites in parallel: Using tornado codes to speed up downloads. In IEEE INFOCOM, pages 275-283, New York, NY, March 1999.

[4] P. M. Chen, E. K. Lee, G. A. Gibson, R. H. Katz, and D. A. Patterson. RAID: High-performance, reliable secondary storage. ACM Computing Surveys, 26(2):145-185, June 1994.

[5] G. A. Gibson. Redundant Disk Arrays: Reliable, Parallel Secondary Storage. The MIT Press, Cambridge, Massachusetts, 1992.

[6] G. A. Gibson, L. Hellerstein, R. M. Karp, R. H. Katz, and D. A. Patterson. Failure correction techniques for large disk arrays. In Third International Conference on Architectural Support for Programming Languages and Operating Systems, pages 123-132, Boston, MA, April 1989.

[7] P. J. M. Havinga. Energy efficiency of error correction on wireless systems, 1999.

[8] C. S. Jutla. Encryption modes with almost free message integrity. Lecture Notes in Computer Science, 2045 , 2001.

[9] J. Kubiatowicz, D. Bindel, Y. Chen, P. Eaton, D. Geels, R. Gummadi, S. Rhea, H. Weatherspoon, W. Weimer, C. Wells, and B. Zhao. Oceanstore: An architecture for global-scale persistent storage. In Proceedings of ACM ASPLOS. ACM, November 2000.

[10] W. Litwin and T. Schwarz. Lh*rs: a high-availability scalable distributed data structure using Reed Solomon codes. In Proceedings of the 2000 ACM SIGMOD International Conference on Management of Data, pages 237-248. ACM Press, 2000.

[11] F. Lowenthal. Linear Algebra with Linear Differential Equations. John Wiley \& Sons, Inc, New York, 1975.

[12] M. Luby. Benchmark comparisons of erasure codes. http://www.icsi.berkeley.edu/ luby/ erasure.html, 2002.

[13] M. Luby, M. Mitzenmacher, and A. Shokrollahi. Analysis of random processes via and-or tree evaluation. In 9th Annual ACM-SIAM Symposium on Discrete Algorithms, January 1998.

[14] M. Luby, M. Mitzenmacher, A. Shokrollahi, D. Spielman, and V. Stemann. Practical loss-resilient codes. In 29th Annual ACM Symposium on Theory of Computing, , pages 150-159, 1997. 
[15] M. Luby, L. Vicisano, J. Gemmell, L. Rizo, M. Handley, and J. Crowcroft. Forward error correction (FEC) building block. IETF RFC 3452 (http : / / www . ietf . org/rfc/rfc3452.txt), December 2002.

[16] M. Luby, L. Vicisano, J. Gemmell, L. Rizo, M. Handley, and J. Crowcroft. The use of forward error correction(FEC) in reliable multicast. IETF RFC 3453 (http: / / www . ietf . org/rfc/rfc3453 . txt), December 2002 .

[17] F.J. MacWilliams and N.J.A. Sloane. The Theory of Error-Correcting Codes, Part I. North-Holland Publishing Company, Amsterdam, New York, Oxford, 1977.

[18] J. S. Plank. Improving the performance of coordinated checkpointers on networks of workstations using RAID techniques. In 15th Symposium on Reliable Distributed Systems, pages 76-85, October 1996.

[19] J. S. Plank. A tutorial on Reed-Solomon coding for fault-tolerance in RAID-like systems. Software - Practice \& Experience, 27(9):995-1012, September 1997.

[20] L. Rizzo. Effective erasure codes for reliable computer communication protocols. ACM SIGCOMM Computer Communication Review, 27(2):24-36, 1997.

[21] L. Rizzo and L. Vicisano. RMDP: an FEC-based reliable multicast protocol for wireless environments. Mobile Computer and Communication Review, 2(2), April 1998.

[22] A. I. T. Rowstron and P. Druschel. Storage management and caching in PAST, a large-scale, persistent peer-topeer storage utility. In Symposium on Operating Systems Principles, pages 188-201, 2001. 